\title{
Effect of Delta Operator on Umbral Composition in Finite
} Operator calculus

\author{
A Maheswaran $^{1^{*}}$, C. Elango, ${ }^{2}$ \\ ${ }^{1,2}$ Department of Mathematics, Cardamom Planters' Association College, Tamilnadu, India. \\ maheswarancpa@gmail.com
}

\section{ABSTRACT}

The main objective of this paper is to propose the matrix representation of umbral composition and investigate the effect of delta operator on umbral composition by using the sequential representation of delta operator in finite operator calculus.

Keywords: Delta operator; Basic polynomial sequences; Sheffer sequences; Umbral Operator; Umbral Composition.

\section{Academic Discipline And Sub-Disciplines}

Mathematics, Umbral Calculus.

\section{SUBJECT CLASSIFICATION}

Umbral Calculus

\section{INTRODUCTION}

The theory of umbral calculus studies the analogies between several sequences of polynomials $p_{n}$ and the monomials $\left\{x^{n} / n \in \mathbb{Z}^{+} \cup\{0\}\right\}$. It is developed in 1970's by G.C.Rota and he linked umbral composition with Finite Operator Calculus. Currently, Umbral Calculus refers to the study of Sheffer sequences, including polynomial sequences of binomial type and Appel sequences.

Emil.C. Popa [3] presents the Taylor formula in Umbral Calculus. Some applications of the Umbral Calculus are discussed in Di.Bucchianico [1]. G.C.Rota [9] serves us a grips introduction and a guide to the growing literature on combinatorics and it contains a detailed study of umbral operator and umbral composition. The goal of Ira M.Gessel [4] is to show,how the classical umbral calculus can be used to proving some interesting formulas not all can easily proved by other methods. Roman M.Steven and G.C.Rota [8] is a detailed study of umbral calculus and applications of combinatorics. A.Maheswaran [6] introduced a new approach to the delta operator as a sequence of real numbers. The characterization of Sheffer umbrace is analyzed in [2].

The aim of the present paper is to propose some results tied to the umbral operator and umbral composition in Umbral Calculus. The rest of the paper is organized in four sections. In the second section, we introduce some basic definitions, operation, notation and theorems from G.C.Rota we need. In the third section, we discussed about the sequential representation of delta operator. Due to the discussion on umbral operator and umbral composition, we propose the matrix representation of umbral composition in fourth section. Finally the effect of delta operator on umbral composition of two basic polynomial sequences are analysed in fifth section.

\section{PRELIMINARIES}

Let $p(x)$ be a polynomial in one variable defined over a field $F$ of characteristic zero and let $\left\{P_{n}(x) / n \in \mathbb{Z}^{+} U\{0\}\right\}$, be a sequence of polynomials, where $p_{n}(x)$ is exactly of degree $n$.

\section{Definition 1.}

i. An operator $E^{a}$ is said to be a shift operator if $E^{a} p(x)=p(x+a)$, for all polynominals $p(x)$ in one variable and for all real ' $a$ ' in the field.

ii. A linear operator $T$ which commutes with all shift operators is called a shift invariant. i.e. $\mathrm{TE}^{\mathrm{a}}=\mathrm{E}^{\mathrm{a}} \mathrm{T}$.

iii. A shift invariant operator $Q$ satisfying that $Q x$ is an non zero constant is called a delta operator.

\section{Definition 2.}

Let $Q$ be the delta operator, $A$ polynomial sequence $p_{n}(x)$ is called sequence of basic polynomials for $Q$ if :

$$
\begin{aligned}
\text { i. } & P_{0}(x)=1 \\
\text { ii. } & P_{n}(0)=0, \text { whenever } n>0 \\
\text { iii. } & Q p_{n}(x)=n p_{n-1}(x)
\end{aligned}
$$

A trivial example for basic polynomial sequence is $\left\{x^{n}\right\}$.

\section{Theorem 1.}

i. Every delta operator has a unique sequence of basic polynomials.

ii. If $Q$ is a delta operator, the $Q a=0$, for every constant ' $a$ '. 
iii. If $p(x)$ is a polynomial of degree $n$, then $Q p(x)$ is a polynomial of degree $n-1$.

Proof. Refer to G-C Rota [3]

By above [i],[ii] and[iii] in Theorem 1, Delta operator Q possesses many of the properties of usual derivative operator D.

\section{Definition 3.}

A polynomial sequence $S_{n}(x)$ is called a Sheffer set or a set of Sheffer polynomials for the delta operator $Q$ if

i. $\quad \mathrm{S}_{0}(\mathrm{x})=\mathrm{C} \neq 0$,

ii. $Q s_{n}(x)=n s_{n-1}(x)$

For example, The monomials $\left\{x^{n}: n=0,1,2,3, \ldots\right\}$, the Abel polynomials, the Hermite polynomials, the laguerre polynomials, the Difference polynomials, the Euler polynomials, the Mott polynomials, the Bell polynomials, the SwissKnife polynomials are very popular Sheffer polynomials.

\section{SEQUENTIAL REPRESENTATION OF DELTA OPERATOR $Q$}

Now we attempt to formulate the delta operator interms of sequence of real numbers. By Theorem (1) and the definition for basic polynomial sequence, we obtain the following Theorem.

Theorem 2. For the monomial $\left\{x^{n}: n \in \mathbb{Z}^{+} U\{0\}\right\}$, and for each $\alpha_{r}(r=1,2,3, \ldots n)$ an arbitrary real numbers,

$$
\mathrm{Q}\left(\mathrm{x}^{\mathrm{n}}\right)=\sum_{r=1}^{n}\left(\begin{array}{l}
n \\
r
\end{array}\right) \mathrm{\alpha}_{\mathrm{r}} \mathrm{x}^{\mathrm{n}-\mathrm{r}}
$$

\section{Proof.}

Taking $Q(x)=\alpha_{1} \neq 0$ and construct $Q\left(x^{2}\right)=c_{0} x+c_{1}$. Since $Q$ is shift invariant, we have

$E^{a} \mathrm{Q}\left(\mathrm{x}^{2}\right)=\mathrm{Q} \mathrm{E}^{\mathrm{a}}\left(\mathrm{x}^{2}\right.$. Solving we get $\mathrm{c}_{0}=2 \alpha_{1}$ and $\mathrm{c}_{1}$ is a new independent constant which may be taken as $\alpha_{2}$. Hence $Q\left(x^{2}\right)=2 \alpha_{1} x+\alpha_{2}$. Thus the theorem is true for $n=1$ and 2 .

Let us assume that the result is true for all $n=k$.

Therefore,

$\mathrm{Q}\left(\mathrm{x}^{\mathrm{k}}\right)=\sum_{r=1}^{\mathrm{k}}\left(\begin{array}{l}\mathrm{k} \\ r\end{array}\right) \alpha_{r} x^{k-r}=\left(\begin{array}{c}\mathrm{k} \\ 1\end{array}\right) \alpha_{1} \mathrm{x}^{\mathrm{k}-1}+\left(\begin{array}{c}\mathrm{k} \\ 2\end{array}\right) \alpha_{2} \mathrm{x}^{\mathrm{k}-2}+\ldots+\left(\begin{array}{c}\mathrm{k} \\ \mathrm{r}\end{array}\right) \alpha_{\mathrm{r}} \mathrm{x}^{\mathrm{k}-\mathrm{r}}+\ldots+\alpha_{\mathrm{k}}$

Since $\left\{x^{n}\right\}$ is a basic polynomial sequence, it satisfies $Q p_{n}(x)=n p_{n-1}(x)$ and hence we have,

$$
\mathrm{Q}\left(\mathrm{x}^{\mathrm{k}}\right)=\mathrm{k} \mathrm{x}^{\mathrm{k}-1}
$$

From (3), we see that the delta operator $Q$ is a usual derivative $D$.

From (2) and (3),

$\left(\begin{array}{l}\mathrm{k} \\ 1\end{array}\right) \alpha_{1} \mathrm{x}^{\mathrm{k}-1}+\left(\begin{array}{l}\mathrm{k} \\ 2\end{array}\right) \alpha_{2} \mathrm{x}^{\mathrm{k}-2}+\ldots+\left(\begin{array}{l}\mathrm{k} \\ \mathrm{r}\end{array}\right) \alpha_{\mathrm{r}} \mathrm{x}^{\mathrm{k}-\mathrm{r}}+\ldots+\alpha_{\mathrm{k}}=\mathrm{k} \mathrm{x}^{\mathrm{k}-1}$

By comparing the corresponding terms, we have $\alpha_{1}=1$ and $\alpha_{\mathrm{j}}=0, \mathrm{j}=2,3, \ldots \mathrm{k}$

Therefore, the result is true for $n=k$ means that

$$
\alpha_{1}=1 \text { and } \alpha_{\mathrm{j}}=0(\mathrm{j}=2,3, \ldots \mathrm{k})
$$

Now we have to show that this result is true for $n=k+1$

$$
Q\left(x^{k+1}\right)=Q\left(x^{k} x\right)=Q\left(x^{k}\right) x+Q(x) x^{k}=(k+1) x^{k}
$$

Thus we have

$$
Q\left(x^{k+1}\right)=(k+1) x^{k}
$$

On other hand, using the property that $Q p_{n}(x)=n p_{n-1}(x)$, we have

$$
Q\left(x^{k+1}\right)=(k+1) p_{k}(x)=(k+1) x^{k}
$$

From the Equations (6) and (7), we conclude that the result is true for all $n=k+1$

Thus we proved the Theorem (2).

The following Table (1) contains first few polynomials $Q\left(x^{n}\right)$, for each degree $n$. 


\begin{tabular}{|l|l|}
\hline$n$ & $Q\left(x^{n}\right)$ \\
\hline 1 & $\alpha_{1}$ \\
\hline 2 & $2 \alpha_{1} x+\alpha_{2}$ \\
\hline 3 & $3 \alpha_{1} x^{2}+3 \alpha_{2} x+\alpha_{3}$ \\
\hline 4 & $4 \alpha_{1} x^{3}+6 \alpha_{2} x^{2}+4 \alpha_{3} x+\alpha_{3}$ \\
\hline 5 & $5 \alpha_{1} x^{4}+10 \alpha_{2} x^{3}+10 \alpha_{3} x^{2}+5 \alpha_{4} x+\alpha_{5}$ \\
\hline 6 & $6 \alpha_{1} x^{5}+15 \alpha_{2} x^{4}+20 \alpha_{3} x^{3}+15 \alpha_{4} x^{2}+6 \alpha_{5} x+\alpha_{6}$ \\
\hline
\end{tabular}

Here, $Q\left(x^{n}\right)$ has $n$ independent parameters, $\alpha_{i},(i=1,2,3, \ldots . n)$. These parameters are unique. The characterization of delta operator is determined by the values of $\alpha_{i}^{\prime}$ ( $(i=1,2,3, \ldots n)$. To study the delta operator $Q$, we need analyse only this sequence (1) Theorem (2). This sequential representation of delta operator plays a vital role in deriving many interesting results for basic polynomial sequences and seffer sequences. In next section, we study more about umbral operator and umbral composition.

\section{MATRIX REPRESENTATION FOR UMBRAL COMPOSITION}

The study of umbral composition starts from a classical operator namely 'umbral operator'.

\section{Definition 4.}

An operator $T$ is said to be umbral operator if $T$ maps some basic polynomials $p_{n}(x)$ into another basic polynomials sequence $q_{n}(x)$.

In another words, $T$ is umbral operator if $\operatorname{Tp}_{n}(x)=q_{n}(x)$, where $p_{n}(x)$ and $q_{n}(x)$ are basic polynomials sequences.

Note that an umbral operator is not shift invariant.

Definition 5. The umbral composition of two polynomial sequences $a_{n}(x)=\sum_{k=0}^{n} a_{n k} x^{k}$ and $b_{n}(x)$ is the sequence of polynomials $c_{n}(x)$ defined by

$$
\mathrm{c}_{\mathrm{n}}(\mathrm{x})=\sum_{k=0}^{n} \mathrm{a}_{\mathrm{nk}} \mathrm{b}_{\mathrm{k}}(\mathrm{x})
$$

The notation for umbral composition is given by

$$
c_{n}(x)=a_{n}(b(x))
$$

The umbral composition is evidently associative but not commutative. Infact, simple polynomial sequence form a group under umbral composition, the identity element being $\left\{x^{n}\right\}$. The following results are connected with umbral operator, delta operator and basic polynomials sequence.

\section{Theorem 3.}

Let $\mathrm{T}$ be an umbral operator, then

1. $\mathrm{T}^{-1}$ exists.

2. If $Q$ is a delta operator, then $\mathrm{TQT}^{-1}$ is also a delta operator.

3. T maps every sequence of basic polynomials into a sequence of basic polynomials.

Proof. Refer to G.C.Rota [9].

The next result determines the operators corresponding to the umbral composition.

\section{Theorem 4.}

Let $S_{n}(x)$ and $t_{n}(x)$ be Sheffer sets relative to the delta operator $Q$ and $P$, and to the invertible shift-invatiant operator $S$ and $T$, respectively. Let $q_{n}(x)$ and $p_{n}(x)$ be the basic sets for $Q$ and $P$, and let the indicators of $S, Q$ and $P$ be

$S=s(D), Q=q(D)$ and $P=p(D)$

where $s(t), q(t)$ and $p(t)$ are formal power series. Define $r_{n}(x)$ to be the umbral composition of $s_{n}(x)$ and $t_{n}(x)$, in symbol,

$r_{n}(x)=s_{n}(t(x))$,

then $r_{n}(x)$ is a Sheffer set relative to the shift-invariant operator

$T s(P)=t(d) s(p(D))$ 
and the delta operator

$q(p(D))$,

having as basic set the sequence

$q_{n}(p(x))$.

Proof. See [9]

An umbral interpretation of functional composition of formal power series is discussed in [5]. Now we discuss the general form of umbral composition in matrix form.

Let $\mathrm{a}_{\mathrm{n}}(\mathrm{x})=\sum_{k=0}^{n} \mathrm{a}_{\mathrm{nk}} \mathrm{x}^{\mathrm{k}}$ and $\mathrm{b}_{\mathrm{n}}(\mathrm{x})=\sum_{k=0}^{n} \mathrm{~b}_{\mathrm{nk}} \mathrm{x}^{\mathrm{k}}$

Then by equation (8), the umbral composition of $a_{n}(x)$ and $b_{n}(x)$ is given by :

$c_{n}(x)=\sum_{k=o}^{n} a_{n k} b_{k k} x^{k}$

In other words,

$c_{0}(x)=a_{n 0} b_{00} x^{0}+0 x^{1}+0 x^{2}+0 x^{3}+\cdots+0 x^{n}$

$c_{1}(x)=a_{10} b_{00} x^{0}+a_{11} b_{11} x^{1}+0 x^{2}+\cdots+0 x^{n}$

$c_{2}(x)=a_{20} b_{00} x^{0}+a_{21} b_{11} x^{1}+a_{22} b_{22} x^{2}+\cdots+0 x^{n}$

$c_{n}(x)=a_{n 0} b_{00} x^{0}+a_{n 1} b_{11} x^{1}+a_{n 2} b_{22} x^{2}+\cdots+a_{n n} b_{n n} x^{n}$

Hence the general form of umbral composition can be written in matrix form as,

$$
\left[\begin{array}{l}
c_{0}(x) \\
c_{1}(x) \\
c_{2}(x) \\
c_{3}(x) \\
\vdots \\
c_{n}(x)
\end{array}\right]=\left[\begin{array}{ccccccc}
a_{00} b_{00} & 0 & 0 & 0 & 0 & \cdots & 0 \\
a_{10} b_{00} & a_{11} b_{11} & 0 & 0 & 0 & \cdots & 0 \\
a_{20} b_{00} & a_{21} b_{11} & a_{22} b_{22} & 0 & 0 & \cdots & 0 \\
a_{30} b_{00} & a_{31} b_{11} & a_{32} b_{22} & a_{33} b_{33} & 0 & \cdots & 0 \\
\vdots & \vdots & \vdots & \vdots & \vdots & \vdots & \vdots \\
a_{n 0} b_{00} & a_{n 1} b_{11} & a_{n 2} b_{22} & a_{33} b_{33} & 0 & \cdots & a_{n n} b_{n n}
\end{array}\right]\left[\begin{array}{c}
x_{0} \\
x_{1} \\
x_{2} \\
x_{3} \\
\vdots \\
x_{n}
\end{array}\right]
$$

Thus the general form of umbral composition is an $(n+1) X(n+1)$ lower triangular matrix, whose diagonal elements are not equal to zero and it can be expressed as

$\mathrm{C}_{\mathrm{k}}(\mathrm{x})=\sum_{k=0}^{n} \mathrm{~A}_{\mathrm{kj}} \mathrm{x}_{\mathrm{k}}$

Where

\section{EFFECT OF DELTA OPERATOR ON UMBRAL COMPOSITION}

If $a_{n}(x)$ is a polynomial sequence, then there is a unique linear operator $L$ such that $L\left(x^{n}\right)=a_{n}(x)$, we say that $L$ is the umbral representation of the sequence $a_{n}(x)$.

Let $b_{n}(x)=x^{n}, n \in \mathbb{Z}^{+} U\{0\}$.

It satisfies $b_{0}(x)=1$ and $b_{n}(0)=0$, whenever $n>0$. Hence it is a basic set and also Sheffer. 
The first few polynomials are $b_{1}(x)=x, b_{2}(X)=x^{2}, b_{3}(X)=x^{3}, b_{4}(x)=x^{4}$ and so on.

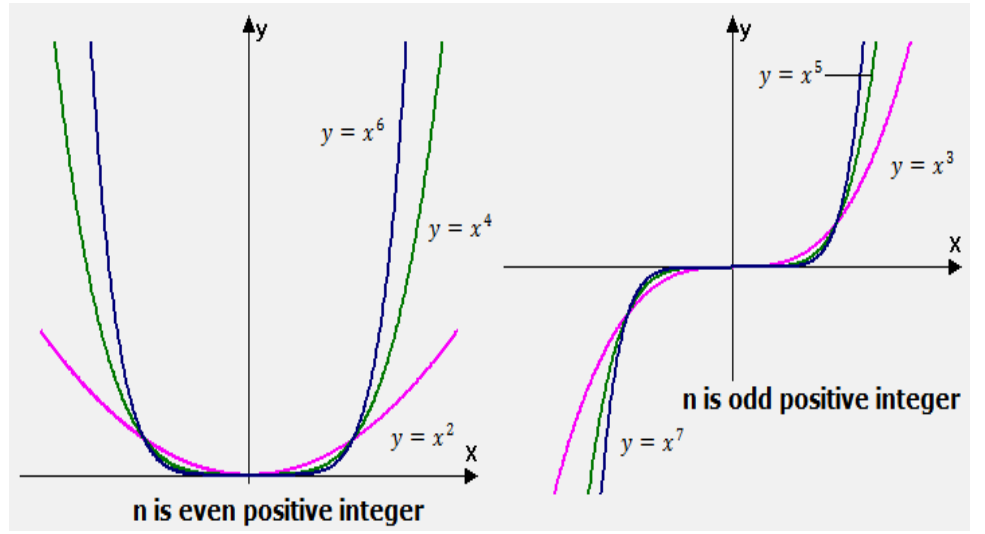

Fig 1: Monomials $\mathbf{b}_{\mathbf{n}}(\mathbf{x})=\left\{\mathbf{x}^{\mathbf{n}} / \mathbf{n} \in \mathbb{Z}^{+} \boldsymbol{U}\{\mathbf{0}\}\right\}$

From the property $Q b_{n}(x)=n b_{n-1}(x)$ and Table (1), we obtain the following proposition.

\section{Proposition 1.}

For the Monomials $b_{n}(x)=\left\{x^{n} / n \in \mathbb{Z}^{+} U\{0\}\right\}$, the characterization of delta operator being $\alpha_{1}=1$, and $\alpha_{r}=0$ for all $r \geq 2$.

Proof: see [9].

Take $a_{n}(x)=\sum_{k=0}^{n} a_{n k} x^{k}$ and $b_{n}(x)=x^{n}$

Then the umbral composition $c_{n}(x)$ of polynomials sequences $a_{n}(x)=a_{n k} x^{k}$ and $b_{n}(x)=x^{n}$ is written in the matrix form as:

$$
\left[\begin{array}{l}
c_{0}(x) \\
c_{1}(x) \\
c_{2}(x) \\
c_{3}(x) \\
\vdots \\
c_{n}(x)
\end{array}\right]=\left[\begin{array}{ccccccc}
a_{00} & 0 & 0 & 0 & 0 & \cdots & 0 \\
a_{10} & a_{11} & 0 & 0 & 0 & \cdots & 0 \\
a_{20} & a_{21} & a_{22} & 0 & 0 & \cdots & 0 \\
a_{30} & a_{31} & a_{32} & a_{33} & 0 & \cdots & 0 \\
\vdots & \vdots & \vdots & \vdots & \vdots & \vdots & \vdots \\
a_{n 0} & a_{n 1} & a_{n 2} & a_{n 3} & a_{n 4} & \cdots & a_{n n}
\end{array}\right]\left[\begin{array}{l}
x_{0} \\
x_{1} \\
x_{2} \\
x_{3} \\
\vdots \\
x_{n}
\end{array}\right]
$$

Now we discuss the effect of delta operator on umbral composition of polynomials set.

The value from the Table 1 and operating $Q$ on the above matrix, we get

$Q c_{0}(x)=0$

$\mathrm{Qc}_{1}(\mathrm{x})=\mathrm{a}_{11} \mathrm{\alpha}_{1}$

$\mathrm{Qc}_{2}(\mathrm{x})=\mathrm{a}_{1}\left(\mathrm{a}_{21}+2 \mathrm{a}_{22} \mathrm{x}\right)+\mathrm{a}_{22} \mathrm{a}_{2}$

$$
=\alpha_{1} \sum_{r=1}^{2}\left(\begin{array}{l}
r \\
1
\end{array}\right) a_{2 r} x^{r-1} a_{2 r} x^{r-1}+a_{22} \alpha_{2}
$$

$\mathrm{Qc}_{3}(\mathrm{x})=\mathrm{a}_{1}\left(\mathrm{a}_{31}+2 \mathrm{a}_{32} \mathrm{x}+3 \mathrm{a}_{33} \mathrm{x} 2\right)+\mathrm{a}_{2}\left(\mathrm{a}_{32}+3 \mathrm{a}_{33} \mathrm{x}\right)+\mathrm{a}_{3} \mathrm{a}_{33}=\mathrm{a}_{1} \sum_{r=1}^{3}\left(\begin{array}{l}r \\ 1\end{array}\right) a_{3 r} x^{r-1}+\mathrm{a}_{2} \sum_{r=2}^{3}\left(\begin{array}{l}r \\ 2\end{array}\right) a_{3 r} x^{r-2}+\mathrm{a}_{3} \mathrm{a}_{33}$

$$
\begin{aligned}
Q_{c}(x)= & a_{1}\left(a_{n 1}+2 a_{n 2} x+3 a_{n 3} x^{2}+\ldots .+{ }_{n} C_{1} a_{n n} x^{n-1}\right) \\
& +a_{2}\left(a_{n 2}+3 a_{n 3} x+12 a_{n 4} x^{2}+\ldots .+{ }_{n} C_{2} a_{n n} x^{n-2}\right) \\
& +a_{3}\left(a_{n 3}+4 a_{n 4} x+20 a_{n 5} x^{2}+\ldots .+{ }_{n} C_{3} a_{n n} x^{n-3}\right) \\
& +\ldots .+a_{n} a_{n n} .
\end{aligned}
$$




$$
\begin{aligned}
\mathrm{Qc}_{\mathrm{n}}(\mathrm{x})=\mathrm{\alpha}_{1} & \sum_{r=1}^{n}\left(\begin{array}{l}
r \\
1
\end{array}\right) a_{n r} x^{r-1}+\mathrm{\alpha}_{2} \sum_{r=2}^{n}\left(\begin{array}{l}
r \\
2
\end{array}\right) a_{n r} x^{r-2}+\alpha_{3} \sum_{r=3}^{n}\left(\begin{array}{l}
r \\
3
\end{array}\right) a_{n r} x^{r-3} \\
& +\ldots+\alpha_{\mathrm{n}} \mathrm{a}_{\mathrm{nn} .}
\end{aligned}
$$

Thus the effect of delta $\mathrm{Q}$ on the umbral composition of $\sum_{k=0}^{n} a_{n k} x^{k}$ and $x^{n}$ can be written in the matrix from as :

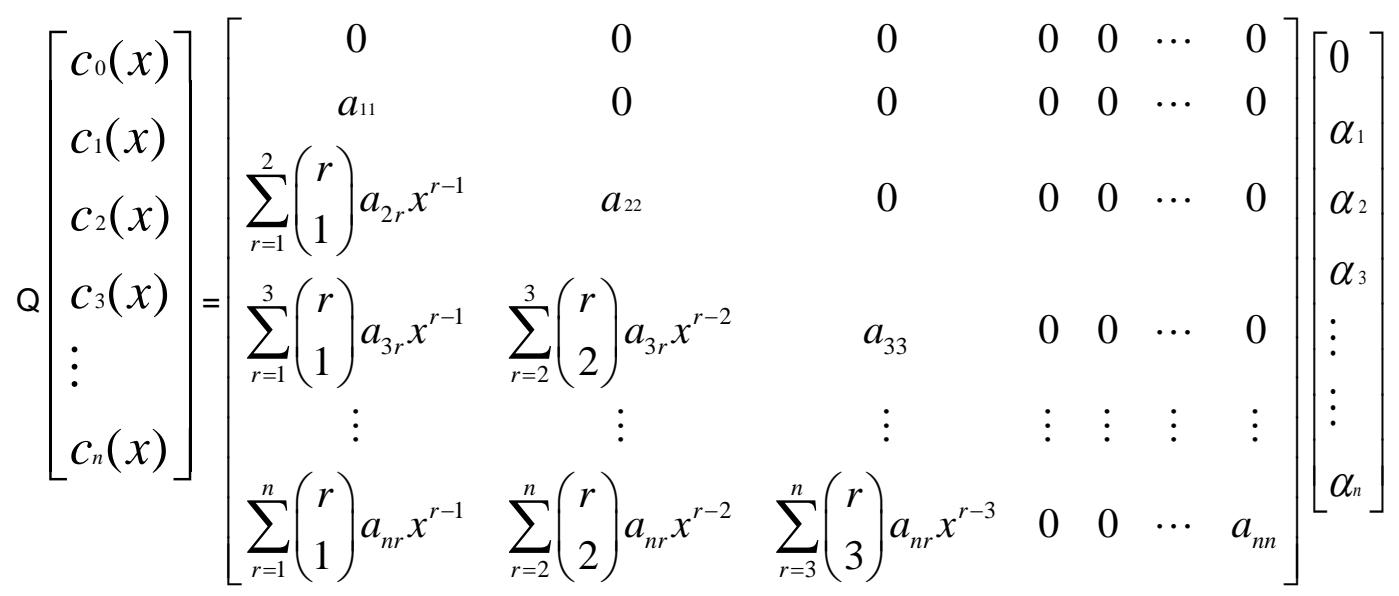

Unbral Calculus is a powerful tool for dealing with recurrences. Recurrences play an important role in the theory of banks in signal processing. An important property of the Umbral Calculus is that it has its own generalization of Lagrange's inversion formula. Thus we find many papers in which new forms of Lagrange's inversion formula is derived using Umbral Calculus.

\section{STATUS AND FURTHER DIRECTIONS}

The present attempt is to study the effect of the delta operator on umbral composition of sheffer sequence via sequential representation in equation (1) Theorem (2). In this study, we consider the monomials $\left\{x^{n}\right\}$ for bn (x). The same theory may be extended to other popular Sheffer polynomials such as falling factorial $[x]_{n}$ or raising factorial $[x]^{n}$ instead of the monomials $\left\{x^{n}\right\}$.

\section{References}

[1] Di Bucchianico, A and Loeb, A Selected Survey of Umbral Calculus, Technische Universiteit Eindhoven, Department of Technology Management, (2000).

[2] Di. Nardo, E, Niederhansen $\mathrm{H}$ and Senato, D, A symbolic handling of Sheffer polynomials, Annali Di Mathematica Pura Ed Applicata, Springer, September 2011.

[3] Email C.Popa. On an expansion theorem in the finite operator calculus of G.C.Rota, General Mathematics, Vol. 16, No. 4 (2008), 149-154.

[4] Ira M. Gessel, Applications of the Classical Umbral Calculus, Research article supported by NSF grand DMS9972648, (2001)

[5] JianhongShen, G.C. Rota and D. Tailor, All polynomials of binomial type are represented by Abel polynomials, MIT, Cambridge, USA, (1997).

[6] Maheswaran A and Elango, C ,Sequential Representation of Delta Operator in Finite Operator Calculus, British Journal of Mathematics and Computer Science, 14(2): 1-11, 2016, DOI: 10.9734/BJMCS/2016/23323.

[7] Maheswaran A, and Elango, C, On new identities for Basic Polynomials Sequences in Finite Operator Calculus, British Journal of Mathematics and Computer Science, 16(3): 2016, 1-11, DOI: 10.9734/BJMCS/2016/24896.

[8] Roman, Steven and Rota G.C. , TheUmbral Calculus, Academic Press, New York London, Feb 1978

[9] Rota G.C., Finite Operator Calculus, Academic Press, London, 1975. 